\title{
Cartesian Composition in Bipolar Fuzzy Finite State Machines
}

\author{
S. Subramaniyan \\ Assistant Professor \\ Mathematics section,FEAT \\ Annamalai University \\ Chidambaram \\ Tamilnadu, India
}

\author{
M. Rajasekar \\ Assistant Professor \\ Mathematics section,FEAT \\ Annamalai University \\ Chidambaram \\ Tamilnadu, India
}

\begin{abstract}
In this paper we introduce Cartesian composition in bipolar fuzzy finite state machines and study their properties.
\end{abstract}

\section{Keywords:}

Bipolar Fuzzy finite state machines, Retrievability, Separability, Cartesian composition, Connectivity.

\section{INTRODUCTION}

The theory of fuzzy set was introduced by L.A. Zadeh in 1965 [9]. The mathematical formulation of a fuzzy automaton was first proposed by W.G. Wee in 1967 [8]. E.S. Santos 1968 [7] proposed fuzzy automata as a model of pattern recognition.

J. N. Mordeson and D.S. Malik gave a detailed account of fuzzy automata and languages in their book 2002 [6]. Fuzzy sets are kind of useful mathematical structure to represent a collection of objects whose boundary is vague. There are several kinds of fuzzy set extensions in the fuzzy set theory, for example, intuitionistic fuzzy sets, interval-valued fuzzy sets etc. Bipolar-valued fuzzy sets, which are introduced by Lee [4, 5], are an extension of fuzzy sets whose membership degree range is enlarged from the interval $[0,1]$ to $[-1,1]$. In [2], Y.B. Jun and J. Kavikumar introduced bipolar fuzzy finite state machines, a bipolar successor, a bipolar exchange property.

In this paper, we introduced Cartesian composition in bipolar fuzzy finite state machines with example and discuss their properties.

\section{BASIC DEFINITIONS}

\subsection{Definition [10]}

Let $X$ denote a universal set. Then a fuzzy set $A$ in $X$ is set of ordered pairs:

$A=\left\{\left(x, \mu_{A}(x) \mid x \in X\right\}\right.$,

$\mu_{A}(x)$ is called the membership function or grade of membership of $x$ in $A$ which maps $X$ to the membership space $[0,1]$.

\subsection{Definition [3]}

A finite fuzzy automata is a system of 3 tuples, $M=\left(Q, X, f_{M}\right)$, where,

$Q$-set of states $\left\{q_{1}, q_{2}, \ldots, q_{n}\right\}$

$\Sigma$-alphabets (or) input symbols

$f_{M}$-function from $Q \times X \times Q \rightarrow[0,1]$

$f_{M}\left(q_{i}, \sigma, q_{j}\right)=\mu,[0 \leq \mu \leq 1]$ means when $M$ is in state $q_{i}$ and reads the input $\sigma$ will move to the state $q_{j}$ with weight function $\mu$.

\subsection{Definition [2]}

A bipolar fuzzy finite state machine (bffsm, for short) is a triple $M=(Q, X, \varphi)$, where $Q$ and $X$ are finite nonempty sets, called the set of states and the set of input symbols, respectively and $\varphi=\left\langle\varphi^{-}, \varphi^{+}\right\rangle$is a bipolar fuzzy set in $Q \times X \times Q$.

Let $X^{*}$ denote the set of all words of elements of $X$ of finite length. Let $\lambda$ denote the empty word in $X^{*}$ and $|x|$ denote the length of $x$ for every $x \in X^{*}$.

\subsection{Definition [2]}

Let $M=(Q, X, \varphi)$ be a bffsm. Define a bipolar fuzzy set $\varphi_{*}=$ $\left\langle\varphi_{*}^{+}, \varphi_{*}^{-}\right\rangle$in $Q \times X^{*} \times Q$ by

$$
\begin{aligned}
& \varphi_{*}^{-}(q, \lambda, p)= \begin{cases}-1 & \text { if } q=p \\
0 & \text { if } q \neq p\end{cases} \\
& \varphi_{*}^{+}(q, \lambda, p)= \begin{cases}1 & \text { if } q=p \\
0 & \text { if } q \neq p\end{cases}
\end{aligned}
$$

$\varphi_{*}^{-}(q, x a, p)=\inf _{r \in Q}\left[\varphi_{*}^{-}(q, x, r) \vee \varphi_{*}^{-}(r, a, p)\right]$

$\varphi_{*}{ }^{+}(q, x a, p)=\sup _{r \in Q}\left[\varphi_{*}^{+}(q, x, r) \wedge \varphi_{*}^{+}(r, a, p)\right] \forall p, q \in$ $Q, x \in X^{*}$ and $a \in X$.

\section{Result}

Let $M=(Q, X, \varphi)$ be a bffsm. Then $\varphi_{*}^{-}(q, x y, p)=\inf _{r \in Q}\left[\varphi_{*}^{-}(q, x, r) \vee \varphi_{*}^{-}(r, y, p)\right]$ $\varphi_{*}{ }^{+}(q, x y, p)=\sup _{r \in Q}\left[\varphi_{*}{ }^{+}(q, x, r) \wedge \varphi_{*}{ }^{+}(r, y, p)\right] \quad \forall p, q \in Q$ and $x, y \in X^{*}$. 


\subsection{Definition [2]}

Let $M=(Q, X, \varphi)$ be a bffsm and let $p, q \in Q$. Then $p$ is called a immediate successor of $q$ if the following condition holds $\exists a \in X$ such that $\varphi_{*}{ }^{-}(q, a, p)<0$ and $\varphi_{*}{ }^{+}(q, a, p)>0$. We say that $p$ is a successor of $q$ if the following condition holds $\exists x \in X^{*}$ such that $\varphi_{*}{ }^{-}(q, x, p)<0$ and $\varphi_{*}{ }^{+}(q, x, p)>0$.

We denote by $S(q)$ the set of all successors of $q$. For any subset $T$ of $Q$ the set of all successors of $T$ denoted by $S(T)$ is defined to be the set $S(T)=\cup\{S(q) \backslash q \in T\}$

\subsection{Definition}

Let $M=(Q, X, \varphi)$ be a bffsm. Let $T \subseteq Q$. Let $v$ be a bipolar fuzzy subset of $T \times X \times T$ and let $N=(T, X, v)$. The bipolar fuzzy finite state machine $N$ is called a submachine of $M$ if

(i) $v \mid T \times X \times T=v$

(ii) $S_{Q}(T) \subseteq T$.

\subsection{Definition}

Let $M=(Q, X, \varphi)$ be a bffsm. $M$ is said to be retrievable if $\forall q \in Q \forall y \in X^{*}$ if $\exists t \in Q$ such that $\varphi_{*}(q, y, t)=$ $\left[\varphi_{*}{ }^{-}(q, y, t)<0, \varphi_{*}{ }^{+}(q, y, t)>0\right]$, then $\exists x \in X^{*}$ such that $\varphi_{*}(t, x, q)=\left[\varphi_{*}{ }^{-}(t, x, q)<0, \varphi_{*}{ }^{+}(t, x, q)>0\right]$.

\subsection{Definition}

Let $M=(Q, X, \varphi)$ be a bffsm. $M$ is said to be quasi-retrievable if $\forall q \in Q \forall y \in X^{*}$ if $\exists t \in Q$ such that $\varphi_{*}(q, y, t)=$ $\left[\varphi_{*}{ }^{-}(q, y, t)<0, \varphi_{*}{ }^{+}(q, y, t)>0\right]$, then $\exists x \in X^{*}$ such that $\varphi_{*}(q, y x, q)=\left[\varphi_{*}{ }^{-}(q, y x, q)<0, \varphi_{*}{ }^{+}(q, y x, q)>0\right]$, where $\varphi_{*}^{-}(q, y x, q)=\inf _{t \in Q}\left[\varphi_{*}{ }^{-}(q, y, t) \vee \varphi_{*}{ }^{-}(t, x, q)\right]<0$ and $\varphi_{*}^{+}(q, y x, q)=\sup _{t \in Q}\left[\varphi_{*}+(q, y, t) \wedge \varphi_{*}+(t, x, q)\right]>0$.

\subsection{Definition}

Let $M=(Q, X, \varphi)$ be a bffsm. Let $q, r, s \in Q$. Then $r$ and $s$ are said to be $q$-related if $\exists y \in X^{*}$ such that $\varphi_{*}(q, y, r)=$ $\left[\varphi_{*}^{-}(q, y, r)<0, \varphi_{*}^{+}(q, y, r)>0\right]$ and

$\varphi_{*}(q, y, s)=\left[\varphi_{*}{ }^{-}(q, y, s)<0, \varphi_{*}{ }^{+}(q, y, s)>0\right]$.

Note

If $r$ and $s$ are bipolar $q$-related then $r$ and $s$ are said to be $q$-twins if $S(s)=S(r)$.

\subsection{Definition}

Let $M=(Q, X, \varphi)$ be a bffsm. We say that $M$ satisfies the exchange property if the following condition holds:

Let $p, q \in Q$ and let $T \subseteq Q$. Suppose that if $p \in S(T \cup\{q\}), p \notin$ $S(T)$, then $q \in S(T \cup\{p\})$.

\subsection{Definition}

Let $M=(Q, X, \varphi)$ be a bffsm. Let $T \subseteq Q$. Let $v$ be a bipolar fuzzy subset of $T \times X \times T$ and let $N=(T, X, v)$. The bipolar fuzzy finite state machine $N$ is called a submachine of $M$ if

(i) $v \mid T \times X \times T=v$

(ii) $S_{Q}(T) \subseteq T$.

\subsection{Definition}

Let $M=(Q, X, \varphi)$ be a bffsm. $M$ is said to be connected if $M$ has no separated proper submachine.

\subsection{Definition}

Let $M=(Q, X, \varphi)$ be a bffsm. Then $M$ is called strongly connected if $\forall p, q \in Q, p \in S(q)$.

\subsection{Definition}

Let $M=(Q, X, \varphi)$ be a bffsm and let $N=(T, X, v)$ be a submachine of $M . N$ is called proper if $T \neq Q$ and $T \neq \phi$. If $M$ is strongly connected then $M$ has no proper submachine.

\subsection{Definition}

Let $M=(Q, X, \varphi)$ be a bffsm and let $\varphi=\left\langle\varphi^{-}, \varphi^{+}\right\rangle$be a subsystem of $M$. Then $\varphi$ is called cyclic if

$\exists q_{t}^{-} \supseteq \varphi^{-}, q \in Q, t \in[-1,0)$ and

$\exists q_{t}^{+} \subseteq \varphi^{+}, q \in Q, t \in(0,1]$.

Note

Let $M=(Q, X, \varphi)$ be a bffsm. Let $R \subseteq Q$. Then $N=$ $\left(S(R), X, \varphi_{R}\right)$ is a submachine of $M$, where $\varphi_{R}=\left\langle\varphi_{R}^{-}, \varphi_{R}^{+}\right\rangle$, where

$\varphi_{R}^{-}=\left.\varphi^{-}\right|_{S(R) \times X \times S(R)}<0$

$\varphi_{R}^{+}=\left.\varphi^{+}\right|_{S(R) \times X \times S(R)}>0$

\subsection{Definition}

Let $M=(Q, X, \varphi)$ be a bffsm. Let $R \subseteq Q$ and $\left\{N_{i}=\left(Q_{i}, X, \mu_{i}\right) \mid i \in I\right\}$ be the collection of all submachines of $M$ whose state set contains $R$. Define $\langle R\rangle=\cap_{i \in I}\left\{Q_{i} \mid i \in I\right\}$. Then $\langle R\rangle$ is called the submachine generated by $R .\langle R\rangle$ is the smallest submachine of $M$ whose state set contains $R$.

\subsection{Definition}

Let $M=(Q, X, \varphi)$ be a bffsm. Let $T \subseteq Q . T$ is called free if $\forall t \in T, t \notin S(T \mid\{t\})$.

\subsection{Definition}

Let $M_{i}=\left(Q_{i}, X_{i}, \varphi_{i}\right)$ be a bffsm, $i=1,2$ and let $X_{1} \cap X_{2}=\phi$. Let $M_{1} \cdot M_{2}=\left(Q_{1} \times Q_{2}, X_{1} \cup X_{2}, \varphi_{1} \cdot \varphi_{2}\right)$, where

$\left(\varphi_{1} \cdot \varphi_{2}\right)^{-}\left(\left(p_{1}, p_{2}\right), a,\left(q_{1}, q_{2}\right)\right)= \begin{cases}\varphi_{1}^{-}\left(p_{1}, a, q_{1}\right) & \text { if } a \in X_{1} \text { and } p_{2}=q_{2} \\ \varphi_{2}^{-}\left(p_{2}, a, q_{2}\right) & \text { if } a \in X_{2} \text { and } p_{1}=q_{1} \\ 0 & \text { otherwise }\end{cases}$

$\left(\varphi_{1} \cdot \varphi_{2}\right)^{+}\left(\left(p_{1}, p_{2}\right), a,\left(q_{1}, q_{2}\right)\right)= \begin{cases}\varphi_{1}^{+}\left(p_{1}, a, q_{1}\right) & \text { if } a \in X_{1} \text { and } p_{2}=q_{2} \\ \varphi_{2}^{+}\left(p_{2}, a, q_{2}\right) & \text { if } a \in X_{2} \text { and } p_{1}=q_{1} \\ 0 & \text { otherwise }\end{cases}$

$\forall\left(\left(p_{1}, p_{2}\right),\left(q_{1}, q_{2}\right)\right) \in Q_{1} \times Q_{2}, a \in X_{1} \cup X_{2}$. Then $M_{1} \circ M_{2}$ is called the Cartesian composition of $M_{1} \circ M_{2}$.

\subsection{Definition}

Let $M=(Q, X, \varphi)$ be a bffsm. Then $M$ is said to be commutative if $\forall a, b \in X$ and $\forall q, p \in Q$ $\varphi_{*}^{-}(q, a b, p)=\varphi_{*}^{-}(q, b a, p)$ and $\varphi_{*}^{+}(q, a b, p)=\varphi_{*}^{+}(q, b a, p)$ 


\section{PROPERTIES OF CARTESIAN COMPOSITION IN BIPOLAR FUZZY FINITE STATE MACHINES}

\subsection{Theorem}

Let $M_{i}=\left(Q_{i}, X_{i}, \varphi_{i}\right)$ be a bffsm, $i=1,2$ and let $X_{1} \cap X_{2}=\phi$. Let $M_{1} \circ M_{2}=\left(Q_{1} \times Q_{2}, X_{1} \cup X_{2}, \varphi_{1} \circ \varphi_{2}\right)$ be the Cartesian composition of $M_{1} \circ M_{2}$. Then $\forall x \in X_{1}^{*} \cup X_{2}^{*}, x \neq \lambda$.

$\left(\varphi_{1} \cdot \varphi_{2}\right)_{*}^{-}\left(\left(p_{1}, p_{2}\right), x,\left(q_{1}, q_{2}\right)\right)= \begin{cases}\varphi_{1}^{-}\left(p_{1}, x, q_{1}\right) & \text { if } x \in X_{1}^{*} \text { and } p_{2}=q_{2} \\ \varphi_{2}^{-}\left(p_{2}, x, q_{2}\right) & \text { if } x \in X_{2}^{*} \text { and } p_{1}=q_{1} \\ 0 & \text { otherwise }\end{cases}$

$\left(\varphi_{1} \cdot \varphi_{2}\right)_{*}^{+}\left(\left(p_{1}, p_{2}\right), x,\left(q_{1}, q_{2}\right)\right)= \begin{cases}\varphi_{1}^{+}\left(p_{1}, x, q_{1}\right) & \text { if } x \in X_{1}^{*} \text { and } p_{2}=q_{2} \\ \varphi_{2}^{+}\left(p_{2}, x, q_{2}\right) & \text { if } x \in X_{2}^{*} \text { and } p_{1}=q_{1} \\ 0 & \text { otherwise }\end{cases}$

\section{Proof.}

Let $x \in X_{1}^{*} \cup X_{2}^{*}, x \neq \lambda$ and let $|x|=n$. Suppose that $x \in X_{1}^{*}$. Clearly the result is true if $n=1$. Suppose the result is true $\forall y \in X_{1}^{*},|y|=n-1, n>1$. Let $x=a y$ where $a \in X_{1}$ and $y \in X_{1}^{*}$. Now, $\left(\varphi_{1} \cdot \varphi_{2}\right)_{*}^{-}\left(\left(p_{1}, p_{2}\right), a y,\left(q_{1}, q_{2}\right)\right)=$ $\wedge^{\wedge}\left(r_{1}, r_{2}\right) \in Q_{1} \times Q_{2}\left\{\left(\varphi_{1} \cdot \varphi_{2}\right)^{-}\left(\left(p_{1}, p_{2}\right), a,\left(r_{1}, r_{2}\right)\right) \vee\left(\varphi_{1} \cdot \varphi_{2}\right)_{*}^{-}\left(\left(r_{1}, r_{2}\right), y,\left(q_{1}, q_{2}\right)\right)\right\}$ $=\wedge_{r_{1}} \in Q_{1}\left\{\left(\varphi_{1}^{-}\left(p_{1}, a, r_{1}\right) \vee\left(\varphi_{1} \cdot \varphi_{2}\right)_{*}^{-}\left(\left(r_{1}, p_{2}\right), y,\left(q_{1}, q_{2}\right)\right\}\right.\right.$

$= \begin{cases}\wedge_{r_{1} \in Q_{1}}\left\{\left(\varphi_{1}^{-}\left(p_{1}, a, r_{1}\right), \vee \varphi_{1} *^{-}\left(r_{1}, y, q_{1}\right)\right)\right\} & \text { if } p_{2}=q_{2} \\ 0 & \text { otherwise }\end{cases}$

$$
= \begin{cases}\left(\varphi_{1} *^{-}\left(p_{1}, a y, q_{1}\right)\right) & \text { if } p_{2}=q_{2} \\ 0 & \text { otherwise }\end{cases}
$$

Now,

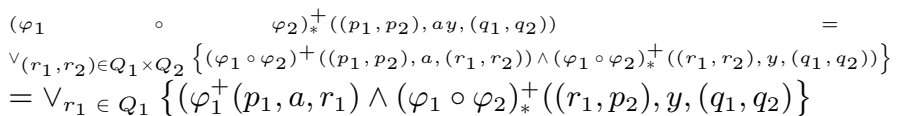

$= \begin{cases}\vee_{r_{1} \in Q_{1}}\left\{\left(\varphi_{1}^{+}\left(p_{1}, a, r_{1}\right), \wedge \varphi_{1} *^{+}\left(r_{1}, y, q_{1}\right)\right)\right\} & \text { if } p_{2}=q_{2} \\ 0 & \text { otherwise }\end{cases}$

$$
= \begin{cases}\left(\varphi_{1} *^{+}\left(p_{1}, a y, q_{1}\right)\right) & \text { if } p_{2}=q_{2} \\ 0 & \text { otherwise }\end{cases}
$$

The result now follows by induction. The proof is similar if $x \in X_{2}^{*}$.

\subsection{Theorem}

Let $M_{i}=\left(Q_{i}, X_{i}, \varphi_{i}\right)$ be a bffsm, $i=1,2$ and let $X_{1} \cap X_{2}=\phi$. Then $\forall x \in X_{1}^{*}, \forall y \in X_{2}^{*}$.

$\left(\varphi_{1} \circ \varphi_{2}\right)_{*}^{-}\left(\left(p_{1}, p_{2}\right), x y,\left(q_{1}, q_{2}\right)\right)=\varphi_{1 *}^{-}\left(p_{1}, x, q_{1}\right) \vee \varphi_{2 *}^{-}\left(p_{1}, y, q_{2}\right)$ $=\left(\varphi_{1} \circ \varphi_{2}\right)_{*}^{-}\left(\left(p_{1}, p_{2}\right), y x,\left(q_{1}, q_{2}\right)\right)$ and

$\left(\varphi_{1} \circ \varphi_{2}\right)_{*}^{+}\left(\left(p_{1}, p_{2}\right), x y,\left(q_{1}, q_{2}\right)\right)=\varphi_{1 *}^{+}\left(p_{1}, x, q_{1}\right) \wedge \varphi_{2 *}^{+}\left(p_{1}, y, q_{2}\right)$ $=\left(\varphi_{1} \circ \varphi_{2}\right)_{*}^{+}\left(\left(p_{1}, p_{2}\right), y x,\left(q_{1}, q_{2}\right)\right)$

$\forall\left(p_{1}, p_{2}\right),\left(q_{1}, q_{2}\right) \in Q_{1} \times Q_{2}$.

Proof. Let $x \in X_{1}^{*}, y \in X_{2}^{*}\left(p_{1}, p_{2}\right),\left(q_{1}, q_{2}\right) \in Q_{1} \times Q_{2}$. If $x=\lambda=y$ then $x y=\lambda$. Suppose $\left(p_{1}, p_{2}\right)=\left(q_{1}, q_{2}\right)$ then $p_{1}=q_{1}$ and $p_{2}=q_{2}$. Hence,

$\left(\varphi_{1} \circ \varphi_{2}\right)_{*}^{-}\left(\left(p_{1}, p_{2}\right), x y,\left(q_{1}, q_{2}\right)\right)=-1=-1 \vee-1=$ $\varphi_{1 *}^{-}\left(p_{1}, x, q_{1}\right) \vee \varphi_{2 *}^{-}\left(p_{1}, y, q_{2}\right)$ $\left(\varphi_{1} \circ \varphi_{2}\right)_{*}^{+}\left(\left(p_{1}, p_{2}\right), x y,\left(q_{1}, q_{2}\right)\right)=1=1 \wedge 1=$ $\varphi_{1 *}^{+}\left(p_{1}, x, q_{1}\right) \wedge \varphi_{2 *}^{+}\left(p_{1}, y, q_{2}\right)$.

If $\left(p_{1}, p_{2}\right) \neq\left(q_{1}, q_{2}\right)$ then either $p_{1} \neq q_{1}$ or $p_{2} \neq q_{2}$.

Thus $\varphi_{1 *}^{-}\left(p_{1}, x, q_{1}\right) \vee \varphi_{2 *}^{-}\left(p_{1}, y, q_{2}\right)=0 \vee 0=0$ and $\varphi_{1 *}^{+}\left(p_{1}, x, q_{1}\right) \wedge \varphi_{2 *}^{+}\left(p_{1}, y, q_{2}\right)=0 \wedge 0=0$

Hence $\left(\varphi_{1} \circ \varphi_{2}\right)_{*}^{-}\left(\left(p_{1}, p_{2}\right), x y,\left(q_{1}, q_{2}\right)\right)=0=\varphi_{1 *}^{-}\left(p_{1}, x, q_{1}\right) \vee$ $\varphi_{2 *}^{-}\left(p_{1}, y, q_{2}\right)$

$\left(\varphi_{1} \circ \varphi_{2}\right)_{*}^{+}\left(\left(p_{1}, p_{2}\right), x y,\left(q_{1}, q_{2}\right)\right)=0=\varphi_{1 *}^{+}\left(p_{1}, x, q_{1}\right) \wedge$ $\varphi_{2 *}^{+}\left(p_{1}, y, q_{2}\right)$.

If $x=\lambda$ and $y \neq \lambda$ or $x \neq \lambda$ and $y=\lambda$ then the result follows by above Theorem. Suppose $x \neq \lambda$ and $y \neq \lambda$. Now

$\left(\varphi_{1} \quad \circ \quad \varphi_{2}\right)_{*}^{-}\left(\left(p_{1}, p_{2}\right), x y,\left(q_{1}, q_{2}\right)\right)$

$\wedge_{r_{1}, r_{2} \in Q_{1} \times Q_{2}}\left\{\left(\varphi_{1} \circ \varphi_{2}\right)_{*}^{-}\left(\left(p_{1}, p_{2}\right), x,\left(r_{1}, r_{2}\right)\right) \vee\left(\varphi_{1} \cdot \varphi_{2}\right)_{*}^{-}\left(\left(r_{1}, r_{2}\right), y,\left(q_{1}, q_{2}\right)\right)\right\}$

$=\wedge r_{1} \in Q_{1}\left\{\left(\varphi_{1} \cdot \varphi_{2}\right)_{*}^{-}\left(\left(p_{1}, p_{2}\right), x,\left(r_{1}, p_{2}\right)\right) \vee\left(\varphi_{1} \circ \varphi_{2}\right)_{*}^{-}\left(\left(r_{1}, p_{2}\right), y,\left(q_{1}, q_{2}\right)\right)\right\}$

$=\varphi_{1 *}^{-}\left(p_{1}, x, q_{1}\right) \vee \varphi_{2 *}^{-}\left(p_{1}, y, q_{2}\right)$

$\quad \quad \quad$ ow, $\quad\left(\begin{array}{lll}\varphi_{1} & \circ & \left.\varphi_{2}\right)_{*}^{+}\left(\left(p_{1}, p_{2}\right), x y,\left(q_{1}, q_{2}\right)\right)\end{array}=\right.$ $\vee_{r_{1}, r_{2} \in Q_{1} \times Q_{2}}\left\{\left(\varphi_{1} \circ \varphi_{2}\right)_{*}^{+}\left(\left(p_{1}, p_{2}\right), x,\left(r_{1}, r_{2}\right)\right) \wedge\left(\varphi_{1} \circ \varphi_{2}\right)_{*}^{+}\left(\left(r_{1}, r_{2}\right), y,\left(q_{1}, q_{2}\right)\right)\right\}$ $\vee_{r_{1} \in Q_{1}}\left\{\left(\varphi_{1} \circ \varphi_{2}\right)_{*}^{+}\left(\left(p_{1}, p_{2}\right), x,\left(r_{1}, p_{2}\right)\right) \wedge\left(\varphi_{1} \cdot \varphi_{2}\right)_{*}^{+}\left(\left(r_{1}, p_{2}\right), y,\left(q_{1}, q_{2}\right)\right)\right\}$ $=\varphi_{1 *}^{+}\left(p_{1}, x, q_{1}\right) \vee \varphi_{2 *}^{+}\left(p_{1}, y, q_{2}\right)$.

Similarly,

$\left(\varphi_{1} \cdot \varphi_{2}\right)_{*}^{-}\left(\left(p_{1}, p_{2}\right), y x,\left(q_{1}, q_{2}\right)\right)=\varphi_{2 *}^{-}\left(p_{1}, y, q_{2}\right) \vee \varphi_{1 *}^{-}\left(p_{1}, x, q_{1}\right)$ $\left(\varphi_{1} \cdot \varphi_{2}\right)_{*}^{+}\left(\left(p_{1}, p_{2}\right), y x,\left(q_{1}, q_{2}\right)\right)=\varphi_{2 *}^{+}\left(p_{1}, y, q_{2}\right) \vee \varphi_{1 *}^{+}\left(p_{1}, x, q_{1}\right)$

\subsection{Theorem}

Let $M_{i}=\left(Q_{i}, X_{i}, \varphi_{i}\right)$ be a bffsm, $i=1,2$ and let $X_{1} \cap X_{2}=\phi$. Then $\forall w \in\left(X_{1} \cup X_{2}\right)^{*}, \exists u \in X_{1}^{*}, v \in X_{2}^{*}$ such that $\left(\varphi_{1} \cdot \varphi_{2}\right)_{*}^{-}\left(\left(p_{1}, p_{2}\right), w,\left(q_{1}, q_{2}\right)\right)$ $\left.\varphi_{1} \cdot \varphi_{2}\right)_{*}^{-}\left(\left(p_{1}, p_{2}\right), u v,\left(q_{1}, q_{2}\right)\right)$ and $\left(\varphi_{1} \cdot \varphi_{2}\right)_{*}^{+}\left(\left(p_{1}, p_{2}\right), w,\left(q_{1}, q_{2}\right)\right)$ $\left(\varphi_{1} \cdot \varphi_{2}\right)_{*}^{+}\left(\left(p_{1}, p_{2}\right), u v,\left(q_{1}, q_{2}\right)\right) \forall\left(\left(p_{1}, p_{2}\right),\left(q_{1}, q_{2}\right)\right) \in Q_{1} \times Q_{2}$.

\section{Proof.}

Let $w \in\left(X_{1} \cup X_{2}\right)^{*}$ and $\left(\left(p_{1}, p_{2}\right),\left(q_{1}, q_{2}\right)\right) \in Q_{1} \times Q_{2}$. If $w=\lambda$ then we can choose $u=\lambda=v$. In this case the result is trivially true. Suppose $w \neq \lambda$. If $w \in X_{1}^{*}$ or $w \in X_{2}^{*}$ then again the result is trivially true. Suppose $w \notin X_{1}^{*}$ and $w \notin X_{2}^{*}$

case 1: If $w=x y, x \in X_{1}^{+}, y \in X_{2}^{+}$then the result follows by above Theorem.

case 2: Suppose $w=x_{1} y x_{2}, x_{1}, x_{2} \in X_{1}^{*}$ and $y \in X_{2}^{*}, x_{i}$ and $y$ are non empty strings, $i=1,2$

Let $u=x_{1}, x_{2} \in X_{1}^{*}$ and $v=y$. Now by above Theorem

$\left(\varphi_{1} \cdot \varphi_{2}\right)_{*}^{-}\left(\left(p_{1}, p_{2}\right), x_{2} y,\left(q_{1}, q_{2}\right)\right)$

$\left(\varphi_{1} \cdot \varphi_{2}\right)_{*}^{-}\left(\left(p_{1}, p_{2}\right), y x_{2},\left(q_{1}, q_{2}\right)\right)$ and

$\left(\varphi_{1} \cdot \varphi_{2}\right)_{*}^{+}\left(\left(p_{1}, p_{2}\right), x_{2} y,\left(q_{1}, q_{2}\right)\right)$

$\left(\varphi_{1} \cdot \varphi_{2}\right)_{*}^{+}\left(\left(p_{1}, p_{2}\right), y x_{2},\left(q_{1}, q_{2}\right)\right)$

$\forall\left(p_{1}, p_{2}\right),\left(q_{1}, q_{2}\right) \in Q_{1} \times Q_{2}$. Thus

$\left(\varphi_{1} \cdot \varphi_{2}\right)_{*}^{-}\left(\left(p_{1}, p_{2}\right), x_{1} y x_{2},\left(q_{1}, q_{2}\right)\right)=$

$\wedge_{r_{1}, r_{2} \in Q_{1} \times Q_{2}}$

$\left\{\left(\varphi_{1} \cdot \varphi_{2}\right)_{*}^{-}\left(\left(p_{1}, p_{2}\right), x_{1},\left(r_{1}, r_{2}\right)\right) \vee\left(\varphi_{1} \cdot \varphi_{2}\right)_{*}^{-}\left(\left(r_{1}, r_{2}\right), y x_{2},\left(q_{1}, q_{2}\right)\right)\right\}$

$\left(\varphi_{1} \cdot \varphi_{2}\right)_{*}^{-}\left(\left(p_{1}, p_{2}\right), x_{1} y x_{2},\left(q_{1}, q_{2}\right)\right)=$

$\wedge_{r_{1}, r_{2} \in Q_{1} \times Q_{2}}$

$\left\{\left(\varphi_{1} \cdot \varphi_{2}\right)_{*}^{-}\left(\left(p_{1}, p_{2}\right), x_{1},\left(r_{1}, r_{2}\right)\right) \vee\left(\varphi_{1} \cdot \varphi_{2}\right)_{*}^{-}\left(\left(r_{1}, r_{2}\right), x_{2} y,\left(q_{1}, q_{2}\right)\right)\right\}$ $=\left\{\left(\varphi_{1} \cdot \varphi_{2}\right)_{*}^{-}\left(\left(p_{1}, p_{2}\right), x_{1} x_{2} y,\left(q_{1}, p_{2}\right)\right)\right\}$

Now, $\left(\varphi_{1} \cdot \varphi_{2}\right)_{*}^{+}\left(\left(p_{1}, p_{2}\right), x_{1} y x_{2},\left(q_{1}, q_{2}\right)\right)=$

$\wedge_{r_{1}, r_{2} \in Q_{1} \times Q_{2}}$

$\left\{\left(\varphi_{1} \cdot \varphi_{2}\right)_{*}^{+}\left(\left(p_{1}, p_{2}\right), x_{1},\left(r_{1}, r_{2}\right)\right) \vee\left(\varphi_{1} \cdot \varphi_{2}\right)_{*}^{+}\left(\left(r_{1}, r_{2}\right), y x_{2},\left(q_{1}, q_{2}\right)\right)\right\}$ $\left(\varphi_{1} \cdot \varphi_{2}\right)_{*}^{+}\left(\left(p_{1}, p_{2}\right), x_{1} y x_{2},\left(q_{1}, q_{2}\right)\right)=$ 
$\wedge_{r_{1}, r_{2} \in Q_{1} \times Q_{2}}$

case 3: $=\left\{\left(\varphi_{1} \cdot \varphi_{2}\right)_{*}^{+}\left(\left(p_{1}, p_{2}\right), x_{1} x_{2} y,\left(q_{1}, p_{2}\right)\right)\right\}$

Suppose $w=y_{1} x y_{2}, y_{1}, y_{2} \in X_{2}^{*}$ and $x \in X_{1}^{*}, y_{i}$ and $x$ are non empty strings, $i=1,2$

Let $v=y_{1}, y_{2} \in X_{2}^{*}$ and $u=x$. The proof is similar to case 2 .

\section{case 4:}

Suppose $w=x_{1} y_{1} x_{2} y_{2}, x_{1}, x_{2} \in X_{1}^{*} y_{1}, y_{2} \in X_{2}^{*}$ and $x_{i}$ and $y_{i}$ are non empty strings, $i=1,2$

Let $u=x_{1} x_{2} \in X_{1}^{*}$ and $v=y_{1} y_{2} \in X_{2}^{*}$. Then

$\left(\varphi_{1} \cdot \varphi_{2}\right)_{*}^{-}\left(\left(p_{1}, p_{2}\right), x_{1} y_{1} x_{2} y_{2},\left(q_{1}, q_{2}\right)\right)=\wedge_{r_{1}, r_{2} \in Q_{1} \times Q_{2}}$

$\left\{\left(\varphi_{1} \circ \varphi_{2}\right) *^{-}\left(\left(p_{1}, p_{2}\right), x_{1},\left(r_{1}, r_{2}\right)\right) \vee\left(\varphi_{1} . \varphi_{2}\right)_{*}^{-}\left(\left(r_{1}, r_{2}\right), y_{1} x_{2} y_{2},\left(q_{1}, q_{2}\right)\right)\right\}$

$=\wedge_{r_{1}, r_{2} \in Q_{1} \times Q_{2}}\left\{\left(\varphi_{1} \circ \varphi_{2}\right)_{*}^{-}\left(\left(p_{1}, p_{2}\right), x_{1},\left(r_{1}, r_{2}\right)\right) \vee\right.$

$\left.\left(\varphi_{1} \circ \varphi_{2}\right)_{*}^{-}\left(\left(r_{1}, r_{2}\right), x_{2} y_{1} y_{2},\left(q_{1}, q_{2}\right)\right)\right\}$ $=\left(\varphi_{1} \circ \varphi_{2}\right)_{*}^{-}\left(\left(p_{1}, p_{2}\right), x_{1} x_{2} y_{1} y_{2},\left(q_{1}, q_{2}\right)\right)$

Now,

$$
=\left(\varphi_{1} \circ \varphi_{2}\right)_{*}^{-}\left(\left(p_{1}, p_{2}\right), u v,\left(q_{1}, q_{2}\right)\right)
$$

$\left(\varphi_{1} \circ \varphi_{2}\right)_{*}^{+}\left(\left(p_{1}, p_{2}\right), x_{1} y_{1} x_{2} y_{2},\left(q_{1}, q_{2}\right)\right)=$

$\vee_{r_{1}, r_{2} \in Q_{1} \times Q_{2}}$

$\left\{\left(\varphi_{1} \circ \varphi_{2}\right) *^{+}\left(\left(p_{1}, p_{2}\right), x_{1},\left(r_{1}, r_{2}\right)\right) \wedge\left(\varphi_{1} \circ \varphi_{2}\right)_{*}^{+}\left(\left(r_{1}, r_{2}\right), y_{1} x_{2} y_{2},\left(q_{1}, q_{2}\right)\right)\right\}$

$=\vee_{r_{1}, r_{2} \in Q_{1} \times Q_{2}}$

$\left\{\left(\varphi_{1} \circ \varphi_{2}\right)_{*}^{+}\left(\left(p_{1}, p_{2}\right), x_{1},\left(r_{1}, r_{2}\right)\right) \wedge\left(\varphi_{1} \circ \varphi_{2}\right)_{*}^{+}\left(\left(r_{1}, r_{2}\right), x_{2} y_{1} y_{2},\left(q_{1}, q_{2}\right)\right)\right\}$

$$
\begin{aligned}
& =\left(\varphi_{1} \circ \varphi_{2}\right)_{*}^{+}\left(\left(p_{1}, p_{2}\right), x_{1} x_{2} y_{1} y_{2},\left(q_{1}, q_{2}\right)\right) \\
& =\left(\varphi_{1} \circ \varphi_{2}\right)_{*}^{+}\left(\left(p_{1}, p_{2}\right), u v,\left(q_{1}, q_{2}\right)\right)
\end{aligned}
$$

\section{case 5:}

Suppose $w=y_{1} x_{1} y_{2} x_{2}, x_{1} x_{2} \in X_{1}^{*}, y_{1}, y_{2} \in X_{2}^{*}$. Let $u=$ $x_{1} x_{2} \in X_{1}^{*}$ and $v=y_{1} y_{2} \in X_{2}^{*}$.

The proof is similar to case 4 .

case 6:

Let $w \in\left(X_{1} \cup X_{2}\right)^{*}$. Then $w=x_{1} y_{1} x_{2} y_{2} \ldots \ldots x_{n} y_{n}$ or $w=$ $y_{1} x_{1} y_{2} x_{2} \ldots \ldots y_{n} x_{n}, x_{i} \in X_{1}^{*}, y_{i} \in X_{2}^{*}, x_{i}$ and $y_{i}$ are nonempty strings $i=1,2, \ldots n-2$.

Let $w=x_{1} y_{1} x_{2} y_{2} \ldots \ldots x_{n} y_{n}$. If $n=0,1$ or 2 the result is true by the previous cases. Now assume that the result is true for $n-1$. Suppose $z=x_{1} y_{1} x_{2} y_{2} \ldots x_{n-1} y_{n-1} \in\left(X_{1} \cup X_{2}\right)^{*}, n \geq 2$.

Let $u_{1}=x_{1} x_{2} \ldots \ldots x_{n-1}, \quad v_{1}=y_{1} y_{2} \ldots \ldots y_{n-1}$

$u=u_{1} x_{n} \quad v=v_{1} y_{n}$

$\left(\varphi_{1} \cdot \varphi_{2}\right)_{*}^{-}\left(\left(p_{1}, p_{2}\right), x_{1} y_{1} x_{2} y_{2} . . x_{n} y_{n},\left(q_{1}, q_{2}\right)\right)=\wedge_{r_{1}, r_{2} \in Q_{1} \times Q_{2}}$

$\left\{\left(\varphi_{1} \circ \varphi_{2}\right) *^{-}\left(\left(p_{1}, p_{2}\right), x_{1} y_{1} . . x_{n-1} y_{n-1},\left(r_{1}, r_{2}\right)\right) \vee\right.$

$\left.\left(\varphi_{1} \circ \varphi_{2}\right)_{*}^{-}\left(\left(r_{1}, r_{2}\right), x_{n} y_{n},\left(q_{1}, q_{2}\right)\right)\right\}$

$=\wedge_{r_{1}, r_{2} \in Q_{1} \times Q_{2}}\left\{\left(\varphi_{1} \circ \varphi_{2}\right)_{*}^{-}\left(\left(p_{1}, p_{2}\right), u_{1} v_{1},\left(r_{1}, r_{2}\right)\right) \vee\right.$

$\left.\left(\varphi_{1} \circ \varphi_{2}\right)_{*}^{-}\left(\left(r_{1}, r_{2}\right), x_{n} y_{n},\left(q_{1}, q_{2}\right)\right)\right\}$

$=\left(\varphi_{1} \circ \varphi_{2}\right)_{*}^{-}\left(\left(p_{1}, p_{2}\right), u_{1} v_{1} x_{n} y_{n},\left(q_{1}, q_{2}\right)\right)$

Now,$$
=\left(\varphi_{1} \circ \varphi_{2}\right)_{*}^{-}\left(\left(p_{1}, p_{2}\right), u_{1} x_{n} v_{1} y_{n},\left(q_{1}, q_{2}\right)\right)
$$

$\left(\varphi_{1} \circ \varphi_{2}\right)_{*}^{+}\left(\left(p_{1}, p_{2}\right), x_{1} y_{1} x_{2} y_{2} \ldots x_{n} y_{n},\left(q_{1}, q_{2}\right)\right)=\vee_{r_{1}, r_{2} \in Q_{1} \times Q_{2}}$

$\left\{\left(\varphi_{1} \circ \varphi_{2}\right) *^{+}\left(\left(p_{1}, p_{2}\right), x_{1} y_{1} \ldots x_{n-1} y_{n-1},\left(r_{1}, r_{2}\right)\right) \wedge\right.$

$\left.\left(\varphi_{1} \circ \varphi_{2}\right)_{*}^{+}\left(\left(r_{1}, r_{2}\right), x_{n} y_{n},\left(q_{1}, q_{2}\right)\right)\right\}$

$=\vee_{r_{1}, r_{2} \in Q_{1} \times Q_{2}}\left\{\left(\varphi_{1} \circ \varphi_{2}\right)_{*}^{+}\left(\left(p_{1}, p_{2}\right), u_{1} v_{1},\left(r_{1}, r_{2}\right)\right)\right.$

$\left.\wedge\left(\varphi_{1} \circ \varphi_{2}\right)_{*}^{+}\left(\left(r_{1}, r_{2}\right), x_{n} y_{n},\left(q_{1}, q_{2}\right)\right)\right\}$

$=\left(\varphi_{1} \circ \varphi_{2}\right)_{*}^{+}\left(\left(p_{1}, p_{2}\right), u_{1} v_{1} x_{n} y_{n},\left(q_{1}, q_{2}\right)\right)$

$=\left(\varphi_{1} \circ \varphi_{2}\right)_{*}^{+}\left(\left(p_{1}, p_{2}\right), u_{1} x_{n} v_{1} y_{n},\left(q_{1}, q_{2}\right)\right)$

The result now follows by induction.

\subsection{Theorem}

Let $M_{i}=\left(Q_{i}, X_{i}, \varphi_{i}\right)$ be a bffsm, $i=1,2$ and let $X_{1} \cap X_{2}=\phi$. Then the Cartesian composition $M_{1} \circ M_{2}$ is cyclic if and only if $M_{1}$ and $M_{2}$ are cyclic.
Proof.

Suppose $M_{1}$ and $M_{2}$ are cyclic, say $Q_{1}=S\left(q_{0}\right)$ and $Q_{2}=S\left(p_{0}\right)$ for some $q_{0} \in Q_{1}, p_{0} \in Q_{2}$. Let $(q, p) \in Q_{1} \times Q_{2}$ Then $\exists x \in X_{1}^{*}$ and $y \in X_{2}^{*}$ such that $\varphi_{1 *}^{-}\left(q_{0}, x, q\right)<0$ and $\varphi_{2 *}^{-}\left(p_{0}, y, p\right)<0$ $\varphi_{1 *}^{+}\left(q_{0}, x, q\right)>0$ and $\varphi_{2 *}^{+}\left(p_{0}, y, p\right)>0$. Thus

$\left(\varphi_{1} \circ \varphi_{2}\right)_{*}^{-}\left(\left(q_{0}, p_{0}\right), x y,(q, p)\right)=\varphi_{1 *}^{-}\left(q_{0}, x, q\right) \vee \varphi_{2 *}^{-}\left(p_{0}, y, p\right)<0$ and

$\left(\varphi_{1} \circ \varphi_{2}\right)_{*}^{+}\left(\left(q_{0}, p_{0}\right), x y,(q, p)\right)=\varphi_{1 *}^{+}\left(q_{0}, x, q\right) \wedge \varphi_{2 *}^{-}\left(p_{0}, y, p\right)>$

Hence $(q, p) \in S\left(q_{0}, p_{0}\right)$. Thus $Q_{1} \times Q_{2}=S\left(q_{0}, p_{0}\right)$. Hence $M_{1} \circ M_{2}$ is cyclic.

\section{Conversely,}

Suppose $M_{1} \circ M_{2}$ is cyclic. Let $Q_{1} \times Q_{2}=S\left(q_{0}, p_{0}\right)$ for some $\left(q_{0}, p_{0}\right) \in Q_{1} \times Q_{2}$. Let $q \in Q_{1}$ and $p \in Q_{2}$. Then $\exists w \in\left(X_{1} \cup X_{2}\right)^{*}$ such that $\left(\varphi_{1} \circ \varphi_{2}\right)_{*}^{-}\left(\left(q_{0}, p_{0}\right), w,(q, p)\right)<0$ and

$\left(\varphi_{1} \circ \varphi_{2}\right)_{*}^{+}\left(\left(q_{0}, p_{0}\right), w,(q, p)\right)>0$. Then by above Theorem, $\exists u \in X_{1}^{*}$ and $v \in X_{2}^{*}$ such that $\varphi_{1 *}^{-}\left(q_{0}, u, q\right) \vee \varphi_{2 *}^{-}\left(p_{0}, v, p\right)=$ $\left(\varphi_{1} \circ \varphi_{2}\right)_{*}^{-}\left(\left(q_{0}, p_{0}\right), w,(q, p)\right)<0$ and

$\varphi_{1 *}^{+}\left(q_{0}, u, q\right) \wedge \varphi_{2 *}^{-}\left(p_{0}, v, p\right)=\left(\varphi_{1} \circ \varphi_{2}\right)_{*}^{+}\left(\left(q_{0}, p_{0}\right), w,(q, p)\right)>$ 0 . Hence $\exists u v \in X_{1}^{*}$ and $v \in X_{2}^{*}$ such that $\varphi_{1 *}^{-}\left(q_{0}, u, q\right)<0$ and $\varphi_{2 *}^{-}\left(p_{0}, v, p\right)<0$.

$\varphi_{1 *}^{+}\left(q_{0}, u, q\right)>0$ and $\varphi_{2 *}^{+}\left(p_{0}, v, p\right)>0$. Thus $q \in S\left(q_{0}\right)$ and $p \in S\left(p_{0}\right)$. Hence $Q_{1}=S\left(q_{0}\right)$ and $Q_{2}=S\left(p_{0}\right)$. Therefore $M_{1}$ and $M_{2}$ are cyclic.

\subsection{Theorem}

Let $M_{i}=\left(Q_{i}, X_{i}, \varphi_{i}\right)$ be a bffsm, $i=1,2$ and let $X_{1} \cap X_{2}=\phi$. Then the Cartesian composition $M_{1} \circ M_{2}$ is retrievable if and only if $M_{1}$ and $M_{2}$ are retrievable.

\section{Proof.}

Suppose that $M_{1}$ and $M_{2}$ are retrievable.

Let $(q, p),(t, s) \in Q_{1} \times Q_{2}$ and $w \in\left(X_{1} \cup X_{2}\right)^{*}$ be such that $\left(\varphi_{1} \cdot \varphi_{2}\right)_{*}^{-}((q, p), w,(t, s))<0$ and $\left(\varphi_{1} \cdot \varphi_{2}\right)_{*}^{+}((q, p), w,(t, s))>$ 0 . Let $w^{*}=u v$ standard form of $w u \in X_{1}^{*}, v \in X_{2}^{*}$. Then $\left(\varphi_{1} \circ \varphi_{2}\right)_{*}^{-}((q, p), w,(t, s))=\left(\varphi_{1} \cdot \varphi_{2}\right)_{*}^{-}((q, p), u v,(t, s))$

$$
=\varphi_{1 *}^{-}(q, u, t) \vee \varphi_{2 *}^{-}(p, v, s)
$$

$\left(\varphi_{1} \circ \varphi_{2}\right)_{*}^{+}((q, p), w,(t, s))=\left(\varphi_{1} \cdot \varphi_{2}\right)_{*}^{+}((q, p), u v,(t, s))$ $=\varphi_{1 *}^{+}(q, u, t) \wedge \varphi_{2 *}^{+}(p, v, s)$. Thus

$\varphi_{1 *}^{-}(q, u, t)<0$ and $\varphi_{2 *}^{-}(p, v, s)<0$

$\varphi_{1 *}^{+}(q, u, t)>0$ and $\varphi_{2 *}^{+}(p, v, s)>0$. Since $M_{1}$ and $M_{2}$ are retrievable, $\exists u^{\prime 6} \in X_{1}^{*}, \quad v^{\prime} \in X_{2}^{*}$ such that $\varphi_{1 *}^{-}\left(t, u^{\prime}, q\right)<0$ and $\varphi_{2 *}^{-}\left(s, v^{\prime}, p\right)<0$

$\varphi_{1 *}^{+}\left(t, u^{\prime}, q\right)>0$ and $\varphi_{2 *}^{+}\left(s, v^{\prime}, p\right)>0$. Thus

$\left(\varphi_{1} \circ \varphi_{2}\right)_{*}^{-}\left((t, s), u^{\prime} v^{\prime},(q, p)\right)<0$

$\left(\varphi_{1} \circ \varphi_{2}\right)_{*}^{+}\left((t, s), u^{\prime} v^{\prime},(q, p)\right)>0$. Hence $M_{1} \circ M_{2}$ is retrievable. Conversely,

Suppose that $M_{1} \circ M_{2}$ is retrievable. Let $q, t \in Q_{1}$ and $y \in X_{1}^{*}$ be such that $\varphi_{1 *}^{-}(q, y, t)<0$ and $\varphi_{1 *}^{+}(q, y, t)<0$. Then $\forall s \in Q_{2}$ $\left(\varphi_{1} \circ \varphi_{2}\right)_{*}^{-}((q, s), y,(t, s))=\varphi_{1 *}^{-}(q, y, t)<0$ and

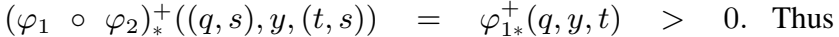
$w \in\left(X_{1} \cup X_{2}\right)^{*}$ such that $\left(\varphi_{1} \circ \varphi_{2}\right)_{*}^{-}((t, s), w,(q, s))<0$ and $\left(\varphi_{1} \circ \varphi_{2}\right)_{*}^{+}((t, s), w,(q, s))>0$.

Let $w^{*}=u v$ standard form of $w u \in X_{1}^{*}, v \in X_{2}^{*}$. Then $0>\left(\varphi_{1} \circ \varphi_{2}\right)_{*}^{-}((t, s), w,(q, s))=\left(\varphi_{1 *}^{-}(t, u, q)\right) \vee\left(\varphi_{2 *}^{-}(s, v, s)\right)$ and

$0<\left(\varphi_{1} \circ \varphi_{2}\right)_{*}^{+}((t, s), w,(q, s))=\left(\varphi_{1 *}^{+}(t, u, q)\right) \wedge\left(\varphi_{2 *}^{-}(s, v, s)\right)$. Hence $M_{1}$ is retrievable. Similarly, $M_{2}$ is retrievable. 


\subsection{Theorem}

Let $M_{i}=\left(Q_{i}, X_{i}, \varphi_{i}\right)$ be a bffsm, $i=1,2$ and let $X_{1} \cap X_{2}=\phi$. Then the Cartesian composition $M_{1} \circ M_{2}$ is connected if and only if $M_{1}$ and $M_{2}$ are connected.

\section{Proof.}

Suppose that $M_{1}$ and $M_{2}$ are connected.

Let $\left(q, q^{\prime}\right),\left(p, p^{\prime}\right) \in Q_{1} \times Q_{2}$, where $q, p \in Q_{1}$ and $q^{\prime}, p^{\prime} \in Q_{2}$. Now $\exists q_{0}, q_{1}, \ldots q_{n} \in Q_{1}, q=q_{0}, p=q_{n}$ and $\exists a_{1}, a_{2}, \ldots . a_{n} \in$ $X_{1}$ such that $\forall i=1,2, \ldots n$ either $\varphi_{1}^{-}\left(q_{i-1}, a_{i}, q_{i}\right)<0$ and $\varphi_{1}^{+}\left(q_{i-1}, a_{i}, q_{i}\right)>0$ or

$\varphi_{1}^{-}\left(q_{i}, a_{i}, q_{i-1}\right)<0$ and $\varphi_{1}^{+}\left(q_{i}, a_{i}, q_{i-1}\right)>0$ and $\exists q_{0}^{\prime}, q_{1}^{\prime}, \ldots q_{m}^{\prime} \in Q_{2}, q^{\prime}=q_{0}^{\prime} p^{\prime}=q_{m}^{\prime}$ and $\exists b_{1}, b_{2}, \ldots b_{m} \in X_{2}$ such that $\forall i=1,2, . . m$ either

$\varphi_{2}^{-}\left(q_{i-1}, b_{i}, q_{i}\right)<0$ and $\varphi_{2}^{+}\left(q_{i-1}, b_{i}, q_{i}\right)>0$ or

$\varphi_{2}^{-}\left(q_{i}, b_{i}, q_{i-1}\right)<0$ and $\varphi_{2}^{+}\left(q_{i}, a_{i}, q_{i-1}\right)>0$.

Consider the sequence of states $\left(q, q^{\prime}\right)=$ $\left(q_{0}, q_{0}^{\prime}\right),\left(q_{1}, q_{0}^{\prime}\right), \ldots\left(q_{n}, q_{0}^{\prime}\right),\left(q_{n}, q_{1}^{\prime}\right), \ldots\left(q_{n}, q_{m}^{\prime}\right)=\left(p, p^{\prime}\right) \in$ $Q_{1} \times Q_{2}$ and the sequence $a_{1}, a_{2}, \ldots a_{n}, b_{1}, b_{2}, \ldots b_{m} \in X_{1} \cup X_{2}$. Then $\forall i=1,2, \ldots n$ either

$\left.\left(\varphi_{1} \circ \varphi_{2}\right)^{-}\left(\left(q_{i}, q_{0}^{\prime}\right), a_{i}, q_{i-1}, q_{0}^{\prime}\right)\right)<0$ and

$\left.\left(\varphi_{1} \circ \varphi_{2}\right)+\left(\left(q_{i}, q_{0}^{\prime}\right), a_{i}, q_{i-1}, q_{0}^{\prime}\right)\right)>0$ or

$\left.\left(\varphi_{1} \circ \varphi_{2}\right)^{-}\left(\left(q_{i-1}, q_{0}^{\prime}\right), a_{i}, q_{i}, q_{0}^{\prime}\right)\right)<0$ and

$\left.\left(\varphi_{1} \circ \varphi_{2}\right)+\left(\left(q_{i-1}, q_{0}^{\prime}\right), a_{i}, q_{i}, q_{0}^{\prime}\right)\right)>0$

$\forall j=1,2, \ldots m$ either

$\left.\left(\varphi_{1} \circ \varphi_{2}\right)^{-}\left(\left(q_{n}, q_{j-1}^{\prime}\right), b_{j}, q_{n}, q_{j}^{\prime}\right)\right)<0$ and

$\left.\left(\varphi_{1} \circ \varphi_{2}\right)^{+}\left(\left(q_{n}, q_{j-1}^{\prime}\right), b_{j}, q_{n}, q_{j}^{\prime}\right)\right)>0$ or

$\left.\left(\varphi_{1} \circ \varphi_{2}\right)^{-}\left(\left(q_{n}, q_{j}^{\prime}\right), b_{j}, q_{n}, q_{j-1}^{\prime}\right)\right)<0$ and

$\left.\left(\varphi_{1} \circ \varphi_{2}\right)^{+}\left(\left(q_{n}, q_{j}^{\prime}\right), b_{j}, q_{n}, q_{j-1}^{\prime}\right)\right)>0$. Hence $\left(q, q^{\prime}\right)$ and $\left(p, p^{\prime}\right)$ are connected.

Conversely,

Suppose that $M_{1} \circ M_{2}$ is connected. Let $q, p \in Q_{1}$ and let $r \in Q_{2}$. If $p=q$ then $p$ and $q$ are connected. Suppose $p \neq q$. then $\exists(q, r)=\left(q_{0}, p_{0}\right),\left(q_{1}, p_{1}\right) \ldots .\left(q_{n}, p_{n}\right)=(p, r) \in Q_{1} \times Q_{2}$ and $a_{1}, a_{2}, \ldots a_{n} \in X_{1} \cup X_{2}$ such that $\forall i=1,2, \ldots n$ either

$\left(\varphi_{1} \circ \varphi_{2}\right)^{-}\left(\left(q_{i-1}, p_{i-1}\right), a_{i},\left(q_{i}, p_{i}\right)\right)<0$ and

$\left(\varphi_{1} \circ \varphi_{2}\right)^{+}\left(\left(q_{i-1}, p_{i-1}\right), a_{i},\left(q_{i}, p_{i}\right)\right)>0$ or

$\left(\varphi_{1} \circ \varphi_{2}\right)^{-}\left(\left(q_{i}, p_{i}\right), a_{i},\left(q_{i-1}, p_{i-1}\right)\right)<0$

$\left(\varphi_{1} \circ \varphi_{2}\right)^{+}\left(\left(q_{i}, p_{i}\right), a_{i},\left(q_{i-1}, p_{i-1}\right)\right)>0$. Clearly, if $q_{i-1} \neq q_{i}$, then $p_{i-1}=p_{i}$ and if $p_{i-1} \neq p_{i}$, then $q_{i-1}=q_{i} \forall i=1,2 \ldots n$.

Let $\left\{q=q_{i 1}, q_{i 2}, \ldots q_{i k}=p\right\}$ be the set of all distinct $q_{i} \in$ $\left\{q_{0}, q_{1}, \ldots q_{n}\right\}$ and let $q_{i 1}, q_{i 2}, \ldots q_{i k}$ be the corresponding $q_{i}^{\prime} s$. Then $q_{i 1}, q_{i 2}, \ldots q_{i k} \in X_{1}$ and $\forall j=1,2, \ldots k$ either

$\left(\varphi_{1} \circ \varphi_{2}\right)^{-}\left(\left(q_{i j-1}, a_{i j}, q_{i j}\right)<0\right.$ and

$\left(\varphi_{1} \circ \varphi_{2}\right)+\left(\left(q_{i j-1}, a_{i j}, q_{i j}\right)>0\right.$ or

$\left(\varphi_{1} \circ \varphi_{2}\right)^{-}\left(\left(q_{i j}, a_{i j}, q_{i j-1}\right)<0\right.$ and

$\left(\varphi_{1} \circ \varphi_{2}\right)^{+}\left(\left(q_{i j}, a_{i j}, q_{i j-1}\right)>0\right.$. Thus $q$ and $p$ are connected. Hence $M_{1}$ is connected. Similarly, $M_{2}$ is connected.

\section{Note}

Let $M_{i}=\left(Q_{i}, X_{i}, \varphi_{i}\right)$ be a bffsm, $i=1,2$ and let $X_{1} \cap X_{2}=\phi$. Then the Cartesian composition $M_{1} \circ M_{2}$ is strongly connected if and only if $M_{1}$ and $M_{2}$ are strongly connected.

\subsection{Theorem}

Let $M_{i}=\left(Q_{i}, X_{i}, \varphi_{i}\right)$ be a bffsm, $i=1,2$ and let $X_{1} \cap X_{2}=\phi$. Then the Cartesian composition $M_{1} \circ M_{2}$ is commutative if and only if $M_{1}$ and $M_{2}$ are commutative.

Proof.

The proof is immediate from Theorem 4.

\subsection{Definition}

Let $M=(Q, X, \varphi)$ be a bffsm. If $M$ is commutative and strongly connected, then $M$ is said to be perfect.

\subsection{Definition.}

Let $M=(Q, X, \varphi)$ be a bffsm. If $M$ is state independent if $\forall q, p \in Q, \forall x, y \in X^{*}$ then $\varphi_{*}^{-}(q, x, p)<0 \Leftrightarrow \varphi_{*}^{-}(q, y, p)<0$ and $\varphi_{*}^{+}(q, x, p)>0 \Leftrightarrow \varphi_{*}^{+}(q, y, p)>0$

\subsection{Theorem}

Let $M_{i}=\left(Q_{i}, X_{i}, \varphi_{i}\right)$ be a bffsm, $i=1,2$ and let $X_{1} \cap X_{2}=\phi$. Then the Cartesian composition $M_{1} \circ M_{2}$ is perfect if and only if $M_{1}$ and $M_{2}$ are perfect.

Proof.

The proof of the Theorem is obvious.

\subsection{Theorem}

Let $M_{i}=\left(Q_{i}, X_{i}, \varphi_{i}\right)$ be a bffsm, $i=1,2$ and let $X_{1} \cap X_{2}=\phi$. Then the Cartesian composition $M_{1} \circ M_{2}$ is state independent if and only if $M_{1}$ and $M_{2}$ are state independent.

\section{Proof.}

Suppose that $M_{1}$ and $M_{2}$ are state independent. Suppose that $\left(\varphi_{1} \circ \varphi_{2}\right)_{*}^{-}\left(\left(q_{1}^{\prime}, q_{2}^{\prime}\right), w_{1},\left(p_{1}^{\prime}, p_{2}^{\prime}\right)\right)<0$

$\left(\varphi_{1} \circ \varphi_{2}\right)_{*}^{+}\left(\left(q_{1}^{\prime}, q_{2}^{\prime}\right), w_{1},\left(p_{1}^{\prime}, p_{2}^{\prime}\right)\right)>0$ and

$\left(\varphi_{1} \circ \varphi_{2}\right)_{*}^{-}\left(\left(q_{1}^{\prime}, q_{2}^{\prime}\right), w_{2},\left(p_{1}^{\prime}, p_{2}^{\prime}\right)\right)<0$

$\left(\varphi_{1} \circ \varphi_{2}\right)_{*}^{+}\left(\left(q_{1}^{\prime}, q_{2}^{\prime}\right), w_{2},\left(p_{1}^{\prime}, p_{2}^{\prime}\right)\right)>0$,

where $\left(q_{1}^{\prime}, q_{2}^{\prime}\right),\left(p_{1}^{\prime}, p_{2}^{\prime}\right) \in Q_{1} \times Q_{2}$ and $w_{1}, w_{2} \in\left(X_{1} \cup X_{2}\right)^{*}$.

Now, $\exists u_{1}, u_{2} \in X_{1}^{+}$and $v_{1}, v_{2} \in X_{2}^{+}$such that

$\left(\varphi_{1} \circ \varphi_{2}\right)_{*}^{-}\left(\left(q_{1}^{\prime}, q_{2}^{\prime}\right), w_{1},\left(p_{1}^{\prime}, p_{2}^{\prime}\right)\right)=\varphi_{1_{*}^{*}}^{-}\left(q_{1}^{\prime}, u_{1}, p_{1}^{\prime}\right) \vee \varphi_{2_{*}^{*}}^{-}\left(q_{2}^{\prime}, v_{1}, p_{2}^{\prime}\right)$

$\left(\varphi_{1} \circ \varphi_{2}\right)_{*}^{+}\left(\left(q_{1}^{\prime}, q_{2}^{\prime}\right), w_{1},\left(p_{1}^{\prime}, p_{2}^{\prime}\right)\right)=\varphi_{1 *}^{+}\left(q_{1}^{\prime}, u_{1}, p_{1}^{\prime}\right) \wedge \varphi_{2 *}^{+}\left(q_{2}^{\prime}, v_{1}, p_{2}^{\prime}\right)$ and $\left(\varphi_{1} \operatorname{circ} \varphi_{2}\right)_{*}^{-}\left(\left(q_{1}^{\prime}, q_{2}^{\prime}\right), w_{2},\left(p_{1}^{\prime}, p_{2}^{\prime}\right)\right)=\varphi_{1 *}^{-}\left(q_{1}^{\prime}, u_{2}, p_{1}^{\prime}\right) \vee \varphi_{2 *}^{-}\left(q_{2}^{\prime}, v_{2}, p_{2}^{\prime}\right)$ $\left(\varphi_{1} \circ \varphi_{2}\right)_{*}^{+}\left(\left(q_{1}^{\prime}, q_{2}^{\prime}\right), w_{2},\left(p_{1}^{\prime}, p_{2}^{\prime}\right)\right)=\varphi_{1 *}^{+}\left(q_{1}^{\prime}, u_{2}, p_{1}^{\prime}\right) \vee \varphi_{2 *}^{+}\left(q_{2}^{\prime}, v_{2}, p_{2}^{\prime}\right)$. Thus

$\varphi_{1 *}^{-}\left(q_{1}^{\prime}, u_{1}, p_{1}^{\prime}\right)<0$ and $\varphi_{1 *}^{+}\left(q_{1}^{\prime}, u_{1}, p_{1}^{\prime}\right)>0$

$\varphi_{2 *}^{-}\left(q_{1}^{\prime}, v_{1}, p_{2}^{\prime}\right)<0$ and $\varphi_{2 *}^{+}\left(q_{1}^{\prime}, v_{1}, p_{2}^{\prime}\right)>0$

$\varphi_{1 *}^{-}\left(q_{1}^{\prime}, u_{2}, p_{1}^{\prime}\right)<0$ and $\varphi_{1 *}^{+}\left(q_{1}^{\prime}, u_{2}, p_{1}^{\prime}\right)>0$

$\varphi_{2 *}^{-}\left(q_{2}^{\prime}, v_{2}, p_{2}^{\prime}\right)<0$ and $\varphi_{2 *}^{+}\left(q_{2}^{\prime}, v_{2}, p_{2}^{\prime}\right)>0$.

Hence $\forall q_{1}, p_{1} \in Q_{1}$,

$\varphi_{1 *}^{-}\left(q_{1}, u_{1}, p_{1}\right)<0 \Leftrightarrow \varphi_{1 *}^{-}\left(q_{1}, u_{2}, p_{1}\right)<0$

$\varphi_{1 *}^{+}\left(q_{1}, u_{1}, p_{1}\right)>0 \Leftrightarrow \varphi_{1 *}^{+}\left(q_{1}, u_{2}, p_{1}\right)>0$ and

$\forall q_{1}, p_{1} \in Q_{1}$,

$\varphi_{2 *}^{-}\left(q_{2}, v_{1}, p_{2}\right)<0 \Leftrightarrow \varphi_{2 *}^{-}\left(q_{2}, v_{2}, p_{2}\right)<0$

$\varphi_{1 *}^{+}\left(q_{2}, v_{1}, p_{2}\right)>0 \Leftrightarrow \varphi_{2 *}^{+}\left(q_{2}, v_{2}, p_{2}\right)>0$. Hence

$\varphi_{1 *}^{-}\left(q_{1}, u_{1}, p_{1}\right) \vee \varphi_{2 *}^{-}\left(q_{2}, v_{1}, p_{2}\right)<0 \Leftrightarrow \varphi_{1 *}^{-}\left(q_{1}, u_{2}, p_{1}\right) \vee$ $\varphi_{1 *}^{-}\left(q_{2}, v_{2}, p_{2}\right)<0$ and

$\varphi_{1 *}^{+}\left(q_{1}, u_{1}, p_{1}\right) \wedge \varphi_{2 *}^{+}\left(q_{2}, v_{1}, p_{2}\right)>0 \Leftrightarrow \varphi_{1 *}^{+}\left(q_{1}, u_{2}, p_{1}\right) \wedge$ $\varphi_{2 *}^{+}\left(q_{2}, v_{2}, p_{2}\right)>0 . \forall q_{1}, p_{1} \in Q_{1}$ and $q_{2}, p_{2} \in Q_{2}$. Thus

$\left(\varphi_{1} \circ \varphi_{2}\right)_{*}^{-}\left(\left(q_{1}, q_{2}\right), w_{1},\left(p_{1}, p_{2}\right)\right)<0 \Leftrightarrow$

$\left(\varphi_{1} \cdot \varphi_{2}\right)_{*}^{-}\left(\left(q_{1}, q_{2}\right), w_{2},\left(p_{1}, p_{2}\right)\right)<0$

$\left(\varphi_{1} \circ \varphi_{2}\right)_{*}^{+}\left(\left(q_{1}, q_{2}\right), w_{1},\left(p_{1}, p_{2}\right)\right)>0 \Leftrightarrow$

$\left(\varphi_{1} \circ \varphi_{2}\right)_{*}^{+}\left(\left(q_{1}, q_{2}\right), w_{2},\left(p_{1}, p_{2}\right)\right)>0$,

$\forall q_{1}, p_{1} \in Q_{1}$ and $q_{2}, p_{2} \in Q_{2}$.

Hence $M_{1} \circ M_{2}$ is state independent.

Conversely,

Suppose that $M_{1} \circ M_{2}$ is state independent. Suppose

$\varphi_{1 *}^{-}\left(q_{1}^{\prime}, u_{1}, p_{1}^{\prime}\right)<0$ and $\varphi_{1 *}^{+}\left(q_{1}^{\prime}, u_{1}, p_{1}^{\prime}\right)>0$ 
$\varphi_{1 *}^{-}\left(q_{1}^{\prime}, u_{2}, p_{1}^{\prime}\right)<0$ and $\varphi_{1 *}^{+}\left(q_{1}^{\prime}, u_{2}, p_{1}^{\prime}\right)>0$ where $u_{1}, u_{2} \in X_{1}^{+}$ and $q_{1}^{\prime}, p_{1}^{\prime} \in Q_{1}$. Then $\forall s \in Q_{2}$

$\left(\varphi_{1} \cdot \varphi_{2}\right)_{*}^{-}\left(\left(q_{1}^{\prime}, s\right), u_{1},\left(p_{1}^{\prime}, s\right)\right)=\varphi_{1 *}^{-}\left(q_{1}^{\prime}, u_{1}, p_{1}^{\prime}\right)<0$ and

$\left(\varphi_{1} \cdot \varphi_{2}\right)_{*}^{+}\left(\left(q_{1}^{\prime}, s\right), u_{1},\left(p_{1}^{\prime}, s\right)\right)=\varphi_{1 *}^{+}\left(q_{1}^{\prime}, u_{1}, p_{1}^{\prime}\right)>0$

$\left(\varphi_{1} \cdot \varphi_{2}\right)_{*}^{-}\left(\left(q_{1}^{\prime}, s\right), u_{2},\left(p_{1}^{\prime}, s\right)\right)=\varphi_{1 *}^{-}\left(q_{1}^{\prime}, u_{2}, p_{1}^{\prime}\right)<0$ and

$\left(\varphi_{1} \cdot \varphi_{2}\right)_{*}^{+}\left(\left(q_{1}^{\prime}, s\right), u_{2},\left(p_{1}^{\prime}, s\right)\right)=\varphi_{1 *}^{+}\left(q_{1}^{\prime}, u_{2}, p_{1}^{\prime}\right)>0$. Thus

$\forall q, p \in Q_{1}, s \in Q_{2}$

$\left(\varphi_{1} \cdot \varphi_{2}\right)_{*}^{-}\left((q, s), v_{1},(p, s)\right)<0 \Leftrightarrow\left(\varphi_{1} \cdot \varphi_{2}\right)_{*}^{-}\left((q, s), u_{2},(p, s)\right)<0$

$\left(\varphi_{1} \cdot \varphi_{2}\right)_{*}^{+}\left((q, s), v_{1},(p, s)\right)>0 \Leftrightarrow\left(\varphi_{1} \cdot \varphi_{2}\right)_{*}^{+}\left((q, s), u_{2},(p, s)\right)>0$.

Hence $\forall q, p \in Q_{1}$,

$\varphi_{1 *}^{-}\left(q, u_{1}, p\right)<0 \Leftrightarrow \varphi_{1 *}^{-}\left(q, u_{2}, p\right)<0$

$\varphi_{1 *}^{+}\left(q, u_{1}, p\right)>0 \Leftrightarrow \varphi_{1 *}^{+}\left(q, u_{2}, p\right)>0$. Thus $M_{1}$ is state independent. Similarly, $M_{2}$ is state independent.

\subsection{Theorem}

Let $M_{i}=\left(Q_{i}, X_{i}, \varphi_{i}\right)$ be a bffsm, $i=1,2$ and let $X_{1} \cap X_{2}=\phi$. Let $N_{i}=\left(T_{i}, X_{i}, \nu_{i}\right)$ be a submachine of $M, i=1,2$. Then $N_{1} \circ N_{2}$ is a submachine of $M_{1} \circ M_{2}$

Conversely, if $N=\left(T_{1} \times T_{2}, X_{1} \cup X_{2}, \nu\right)$ is a submachines $N_{1}$ of $M_{1}$ and $N_{2}$ of $M_{2}$ such that $N=N_{1} \circ N_{2}$.

\section{Proof.}

Let $N_{i}=\left(T_{i}, X_{i}, \nu_{i}\right)$ be a submachine of $M_{i}, i=1,2$. Now, $N_{1} \circ N_{2}=\left(T_{1} \times T_{2}, X_{1} \cup X_{2}, \nu_{1} \circ \nu_{2}\right)$ Let $(r, s) \in S\left(T_{1} \times T_{2}\right)$. Then $\exists w \in\left(X_{1} \cup X_{2}\right)^{*}(p, q) \in T_{1} \times T_{2}$ such that

$\left(\varphi_{1} \cdot \varphi_{2}\right)_{*}^{-}((p, q), w,(r, s))<0$ and

$\left(\varphi_{1} \cdot \varphi_{2}\right)_{*}^{+}((p, q), w,(r, s))>0$

Let $w^{*}=u v$ be the standard form of $w, u \in X_{1}^{*}, v \in X_{2}^{*}$. Now, $\varphi_{1 *}^{-}(p, u, r) \vee \varphi_{2 *}^{-}(q, v, s)=\left(\varphi_{1} \cdot \varphi_{2}\right)_{*}^{-}((p, q), w,(r, s))<0$ and $\varphi_{1 *}^{+}(p, u, r) \vee \varphi_{2 *}^{+}(q, v, s)=\left(\varphi_{1} \cdot \varphi_{2}\right)_{*}^{+}((p, q), w,(r, s))>0$. Hence, $r \in S(p) \subseteq S\left(T_{1}\right)=T_{1}$ and $s \in S(q) \subseteq S\left(T_{2}\right)=T_{2}$. Thus, $(r, s) \in T_{1} \times T_{2}$. Hence, $S\left(T_{1} \times T_{2}\right) \subseteq T_{1} \times T_{2}$. Let $(p, q),(r, s) \in T_{1} \times T_{2}, a \in X_{1} \cup X_{2}$. Now,

$$
\begin{gathered}
\left(\nu_{1} \circ \nu_{2}\right)^{-}((p, q), a,(r, s))= \begin{cases}\nu_{1}^{-}(p, a, r) & \text { if } a \in X_{1} q=s \\
\nu_{2}^{-}(p, a, s) & \text { if } a \in X_{2} p=r \\
0 & \text { otherwise }\end{cases} \\
\left(\nu_{1} \circ \nu_{2}\right)^{+}((p, q), a,(r, s))= \begin{cases}\nu_{1}^{+}(p, a, r) & \text { if } a \in X_{1} q=s \\
\nu_{2}^{+}(p, a, s) & \text { if } a \in X_{2} p=r \\
0 & \text { otherwise }\end{cases} \\
= \begin{cases}\varphi_{1}^{-}(p, a, r) & \text { if } a \in X_{1} q=s \\
\varphi_{2}^{-}(p, a, s) & \text { if } a \in X_{2} p=r \\
0 & \text { otherwise }\end{cases} \\
= \begin{cases}\varphi_{1}^{+}(p, a, r) & \text { if } a \in X_{1} q=s \\
\varphi_{2}^{+}(p, a, s) & \text { if } a \in X_{2} p=r \\
0 & \text { otherwise }\end{cases}
\end{gathered}
$$

$=\left(\varphi_{1} \circ \varphi_{2}\right)^{-}((p, q), a,(r, s))$ and

$=\left(\varphi_{1} \circ \varphi_{2}\right)^{+}((p, q), a,(r, s))$.

Hence, $\left.\left(\varphi_{1} \circ \varphi_{2}\right)^{-}\right|_{\left(T_{1} \times T_{2}\right) \times\left(X_{1} \cup X_{2}\right) \times\left(T_{1} \times T_{2}\right)}=\left(\nu_{1} \circ \nu_{2}\right)^{-}$and $\left.\left(\varphi_{1} \circ \varphi_{2}\right)^{+}\right|_{\left(T_{1} \times T_{2}\right) \times\left(X_{1} \cup X_{2}\right) \times\left(T_{1} \times T_{2}\right)}=\left(\nu_{1} \circ \nu_{2}\right)^{+}$. Thus $\left(\nu_{1} \circ \nu_{2}\right)$ is a submachine of $M_{1} \circ M_{2}$.

Conversely, let $N=\left(T_{1} \times T_{2}, X_{1} \cup X_{2}, \nu\right)$ be a submachine of $M_{1} \circ M_{2}$. Let $\nu_{1}^{-}=\left.\varphi_{1}^{-}\right|_{T_{1} \times X_{1} \times T_{1}}, \quad \nu_{2}^{+}=\left.\varphi_{1}^{+}\right|_{T_{2} \times X_{2} \times T_{2}}, \quad N_{1}=$ $\left(T_{1}, X_{1}, \nu_{1}\right)$, and $N_{2}=\left(T_{2}, X_{2}, \nu_{2}\right)$.
Let $p \in T_{1}, x \in X_{1}^{*}, r \in Q_{1}$ such that $\varphi_{1}^{-} *(p, x, r)<0$ and $\varphi_{1}^{+} *(p, x, r)>0$. Let $t \in T_{2}$. Then,

$\left(\varphi_{1} \circ \varphi_{2}\right)^{-} *((p, t), x,(r, t))=\varphi_{1}^{-} *(p, x, r)<0$ and

$\left(\varphi_{1} \circ \varphi_{2}\right)^{+} *((p, t), x,(r, t))=\varphi_{1}^{+} *(p, x, r)>0$.

Thus, $(r, t) \in S\left(T_{1} \times T_{2}\right)=T_{1} \times T_{2}$. Hence, $r \in T_{1}$ and so $S\left(T_{1}\right) \subseteq T_{1}$. Thus, $N_{1}$ is a submachine of $M_{1}$. Similarly, $N_{2}$ is a submachine of $M_{2}$. Let $(p, q),(r, s) \in T_{1} \times T_{2}$, and $a \in X_{1} \cup X_{2}$. Now,

$\nu^{-}((p, q), a,(r, s))=\left(\varphi_{1} \circ \varphi_{2}\right)^{-}((p, q), a,(r, s))$

$$
\begin{gathered}
= \begin{cases}\varphi_{1}^{-}(p, a, r) & \text { if } a \in X_{1} q=s \\
\varphi_{2}^{-}(p, a, s) & \text { if } a \in X_{2} p=r \\
0 & \text { otherwise }\end{cases} \\
= \begin{cases}\varphi_{1}^{+}(p, a, r) & \text { if } a \in X_{1} q=s \\
\varphi_{2}^{+}(p, a, s) & \text { if } a \in X_{2} p=r \\
0 & \text { otherwise }\end{cases} \\
= \begin{cases}\nu_{1}^{-}(p, a, r) & \text { if } a \in X_{1} q=s \\
\nu_{2}^{-}(p, a, s) & \text { if } a \in X_{2} p=r \\
0 & \text { otherwise }\end{cases} \\
= \begin{cases}\nu_{1}^{+}(p, a, r) & \text { if } a \in X_{1} q=s \\
\nu_{2}^{+}(p, a, s) & \text { if } a \in X_{2} p=r \\
0 & \text { otherwise }\end{cases} \\
=\left(\nu_{1} \circ \nu_{2}\right)^{-}((p, q), a,(r, s)) \text { and }
\end{gathered}
$$

\section{CONCLUSION}

In this paper, we introduce Cartesian composition in bipolar fuzzy finite state machines and discuss their properties.

\section{REFERENCES}

[1] Dubois. D., Prade. H. 1980. Fuzzy Sets and Systems. Mathematics in Science and Engineering, Academic Press, New York, 144.

[2] Jun. Y. B., Kavikumar. J. 2011. Bipolar Fuzzy Finite State Machines. Bull.Malays.Sci.Soc. 34 no. 1: 181-188.

[3] Kandel. A., Lee. S. C. Fuzzy Switching and Automata Theory Applications. Edward Arnold Publishers Ltd, London.

[4] Lee. K. J. 2000. Bipolar-valued fuzzy sets and their operations. Proc. Int. Conf. on Intelligent Technologies, Bangkok, Thailand, 307-312.

[5] Lee. K. J. 2004.Comparison of interval valued fuzzy sets, Intuitionistic fuzzy sets, and bipolar-valued fuzzy sets. J. Fuzzy Logic Intelligent Systems, no.14: 125-129.

[6] Mordeson. J. N., Malik. D. S. 2002. Fuzzy automata and Languages.-Theory and Applications, Chapman \& Hall/ CRC Press.

[7] Santos. E. S. 1968.General formulation of sequential machines. Information and control, no.12: 5-10.

[8] Wee. W. G. 1967. On generalizations of adaptive algorithm and application of the fuzzy sets concept to pattern classification. Ph.D. Thesis Purude University. 
[9] Zadeh. L. A. 1965. Fuzzy sets. Information and control, no. 8: 338-353.

[10] Zimmermann.

$\mathrm{H}$.

J. 1985. Fuzzy set theory and its applications. International Series in Management Science/Operations research, KluwerNijhoff, Boston, MA. 